\title{
High strangeness dibaryons in the extended quark delocalization, color screening model
}

\author{
Hourong Pang \\ Department of Physics, Nanjing University, Nanjing, 210093, P. R. China; \\ Institute of Theoretical Physics, Chinese Academy of Sciences, Beijing, 100080, China \\ Jialun Ping \\ Department of Physics, Nanjing Normal University, Nanjing, 210097, P.R. China; \\ Center for Theoretical Physics, Nanjing University, Nanjing, 210093, P.R. China \\ Fan Wang \\ Center for Theoretical Physics and Department of Physics, \\ Nanjing University, Nanjing, 210093, P. R. China \\ T. Goldman \\ Theoretical Division, Los Alamos National Laboratory, Los Alamos, NM 87545, USA \\ Enguang Zhao \\ Institute of Theoretical Physics, Chinese Academy of Science, Beijing, 100080, China
}




\begin{abstract}
Dibaryon candidates with strangeness $S=-2,-3,-4,-5,-6$ are studied in terms of the extended quark delocalization and color screening model. The results show that there are only a few promising low lying dibaryon states: The $H$ and di- $\Omega$ may be marginally strong interaction stable but model uncertainties are too large to allow any definitive statement. The $S I J=-3,1 / 2,2$ $\mathrm{N} \Omega$ state is $62 \mathrm{MeV}$ lower than the $\mathrm{N} \Omega$ threshold and $24 \mathrm{MeV}$ lower than the $\Lambda \Xi \pi$ threshold. It might appear as a narrow dibaryon resonance and be detectable in the RHIC detector through the reconstruction of the vertex mass of the $\Lambda \Xi$ two body decay. The effects of explicit $K$ and $\eta$ meson exchange have been studied and found to be negligible in this model. The mechanisms of effective intermediate range attraction, $\sigma$ meson exchange and kinetic energy reduction due to quark delocalization are discussed.
\end{abstract}

PACS numbers: 12.39.-x, 14.20.Pt, 13.75.Cs 


\section{INTRODUCTION}

One of the most remarkable achievements of theoretical physics in the past thirty years is the establishment and development of the fundamental theory of the strong interaction - quantum chromodynamics (QCD). Perturbative QCD has been verified by high energy experiments. However, the low energy physics of QCD, such as hadron structure, hadron interactions and the structure of exotic quark-gluon systems, are much harder to calculate directly from QCD. One needs effective theories and phenomenological models in these cases.

Borrowing the idea of quasiparticles from condensed matter and nuclear physics, one can approximately transform the complicated interactions between current quarks into dynamic properties of quasiparticles and what is left to be studied are the residual interactions between quasiparticles. One of the quasiparticles in QCD is the constituent quark. How to dress the current quark to be a constituent quark still poses a theoretical challenge; various effective theories 1, 2, 3] have been developed to derive constituent quarks from QCD. The common point of view is that the dynamical generation of the constituent quark mass is closely related to spontaneous chiral symmetry breaking initiated by the formation of a $q \bar{q}$ condensate in the QCD vacuum.

The constituent quark model has been quite successful in understanding hadron spectroscopy and hadron interactions even though we have not yet derived the constituent quark model directly from QCD. There are also various versions of the constituent quark model based on different effective degrees of freedom. De Rujula, Georgi and Glashow 4] first put forward a quark-gluon coupling model based on constituent quark and gluon effective degrees of freedom. Isgur obtained a good description of hadron spectroscopy based on this model[5]. However, extension of the model to baryon interactions does not reproduce the nucleon-nucleon intermediate and long range interaction.

One modification studied is the addition of Goldstone boson exchange on the quark level[6, 7, 8, 9], in which the short-range part of the interaction is described by the quarkgluon degrees of freedom and the medium and long-range parts are attributed to mesonexchange. This quark gluon-meson exchange hybrid model achieves a quantitative fit of the nucleon-nucleon (NN) and nucleon-hyperon (NY) scattering data.

A different modification of the De Rujula-Georgi-Glashow-Isgur (RGGI) model, the quark delocalization and color screening model (QDCSM) 10], has also been suggested. It main- 
tains the Isgur Hamiltonian for single hadrons but modifies it for baryon-baryon (BB) interactions with two new ingredients.

First, the two center single quark orbital wave function (WF) used in the quark cluster model is replaced by a delocalized quark orbital WF. The introduction of quark delocalization can be viewed either as taking into account the contribution of excited configurations or the distortion of each individual baryon due to their mutual interaction. This straightforward method enlarges the variational Hilbert space. Its advantage is that it permits the six-quark system to choose a more favorable configuration through its own dynamics, while maintaining a tolerable level of computational complexity.

Second, a different parametrization of the confinement interaction is assumed, in which the usual quadratic confinement and a color-screened quadratic confinement are used to parameterize the two body matrix elements of different quark orbits. The introduction of this parametrization is aimed at taking into account some of the nonlinear, nonperturbative properties of QCD which can not be described by two-body quark-quark interactions in multiquark systems, such as the formation of color flux tubes connecting many quarks, the three gluon interaction and the three body instanton interaction.

The QDCSM not only justifies the view of a nucleus as, approximately, a collection of nucleons rather than a single big bag with $3 \mathrm{~A}$ quarks, but it also explains the long standing fact that the nuclear force and molecular forces are similar except for the obvious energy and length scale differences. It is also the model which requires the fewest adjustable parameters to fit the existing BB interaction data 10, 11, 12, 13, 14, 15, 16].

There have been debates on which constituent quark model and which effective degrees of freedom are best to use for hadron structure and interaction studies [17, 18, 19, 20, 21]. In our phenomenological study of BB interactions with three different constituent quark models, we found that even though the QDCSM and the other two models appear to be quite different, they give similar BB interactions in 44 of the 64 lowest BB channels consisting of octet and decuplet baryons 14]. A preliminary analysis of the origin of this surprising similarity has been produced[22]. This result also implies that quark delocalization and color screening, working together, do provide the intermediate range attraction described by meson exchange in other models. On the other hand, the different models do give characteristically different results in some channels. For example, the QDCSM predicts a strong attraction in the $\mathrm{I}=0$, $\mathrm{J}=3$ channel, producing a dibaryon resonance, $d^{*}$, in this channel, while the quark gluon- 
meson exchange hybrid model predicts a strong attraction in the $\mathrm{I}=0, \mathrm{~J}=0 \Omega \Omega$ channel, implying the existence of a strong interaction stable di- $\Omega$. One cannot expect scattering data to become available in these channels to test these model predictions, but the dibaryon states should be detectable to provide a check on whether these model predictions are realistic.

The study of dibaryon states not only checks constituent quark models but also searches for new hadronic matter. The $H$-particle has been assumed to be a six quark system from the very beginning [23] and has been both a theoretical and experimental topic for a long time. The $\mathrm{S}=0, J^{p}=0^{-}$d' dibaryon was assumed to be an $\mathrm{NN} \pi$ system and was a hot topic in the 1990's 13, 24]. We showed that the $S=0, I=0, J=3 d^{*}[10$, 14, 15, 25, 26, 27, 28] is a tightly bound six quark system rather than a loosely bound nuclear-like system of two $\Delta$ 's. An $S=-3, I=1 / 2, J=2 \mathrm{~N} \Omega$ state was proposed as a high strangeness dibaryon candidate[29]. Kopeliovich predicted high strangeness dibaryons, such as the di- $\Omega$ with $S=-6$, using the flavor SU(3) Skyrmion model [30], and Zhang suggested searching for the di- $\Omega$ in ultrarelativistic heavy ion collisions [31]. Lomon predicted a deuteron-like dibaryon resonance using R-matrix theory [32] and measurements at Saclay seem to offer experimental support [33] for its existence.

In this paper, we calculate promising dibaryons with strangeness $S=-2,-3,-4,-5$ using the extended quark-delocalization color-screening model to provide another reference spectrum for strange dibaryons and a possible further check of constituent quark models. The SIJ $=003 d^{*}$ and -600 di- $\Omega$ dibaryons, which we considered previously, are included in the discussion to demonstrate the differences between the predictions of the extended QDCSM and other quark models.

The extended QDCSM is briefly introduced in Section II. In Section III, we present our results. We discuss these results further in Sections IV and V, and conclude in Section VI.

\section{BRIEF DESCRIPTION OF THE EXTENDED QDCSM}

The QDCSM was put forward in the early 90's. Details can be found in Refs. 10, 26, 28]. Although the intermediate range attraction of the $N N$ interaction is reproduced by the combination of quark delocalization and color screening, the effect of the long-range

pion tail is missing in the QDCSM. Recently, the extended QDCSM was developed [15], 
which incorporates this long-range tail by adding $\pi$-exchange with a short-range cutoff. The extended QDCSM not only reproduces the properties of the deuteron well, but also improves agreement with $\mathrm{NN}$ scattering data as compared to previous work [16].

The Hamiltonian of the extended QDCSM, wave functions and the necessary equations used in the current calculation are given below. Here we do not take into account the effect of any tensor forces. The details of the resonating-group method (RGM) have been presented in Refs. 28, 34].

The Hamiltonian for the 3 -quark system is the same as the well known quark potential model, the Isgur model. For the six-quark system, we assume

$$
\begin{aligned}
& H_{6}=\sum_{i=1}^{6}\left(m_{i}+\frac{p_{i}^{2}}{2 m_{i}}\right)-T_{C M}+\sum_{i<j=1}^{6}\left[V_{\text {conf }}\left(r_{i j}\right)+V_{G}\left(r_{i j}\right)+V_{\pi}\left(r_{i j}\right)\right], \\
& V_{G}\left(r_{i j}\right)=\alpha_{s} \frac{\vec{\lambda}_{i} \cdot \vec{\lambda}_{j}}{4}\left[\frac{1}{r_{i j}}-\frac{\pi}{2} \delta\left(\overrightarrow{r_{i j}}\right)\left(\frac{1}{m_{i}^{2}}+\frac{1}{m_{j}^{2}}+\frac{4 \vec{\sigma}_{i} \cdot \vec{\sigma}_{j}}{3 m_{i} m_{j}}\right)\right], \\
& V_{\pi}\left(r_{i j}\right)=\theta\left(r-r_{0}\right) \frac{g_{8}^{2}}{4 \pi} \frac{m_{\pi}^{2}}{12 m_{q}^{2}} \frac{1}{r_{i j}} e^{-m_{\pi} r_{i j}} \vec{\sigma}_{i} \cdot \vec{\sigma}_{j} \vec{\tau}_{i} \cdot \vec{\tau}_{j}, \\
& V_{\text {conf }}\left(r_{i j}\right)=-a_{c} \vec{\lambda}_{i} \cdot \vec{\lambda}_{j} \begin{cases}r_{i j}^{2} & \text { if } i, j \text { occur in the same baryon orbit, } \\
\frac{1-e^{-\mu r_{i j}^{2}}}{\mu} & \text { if } i, j \text { occur in different baryon orbits, }\end{cases} \\
& \theta\left(r_{i j}-r_{0}\right)= \begin{cases}0 & r_{i j}<r_{0} \\
1 & \text { otherwise }\end{cases}
\end{aligned}
$$

where $r_{0}$ is the short range cutoff for pion exchange between quarks. All the symbols have their usual meanings, and the confinement potential $V_{\text {conf }}\left(r_{i j}\right)$ has been discussed in Refs. [15, 28].

The pion potential, $V_{\pi}\left(r_{i j}\right)$, affects only the $u$ and $d$ quarks. We take these to have a common mass, $m_{q}=m_{d}=m_{u}$, ignoring isospin breaking effects as they are small on the scale of interest here.

The quark wave function in a given nucleon (orbit) relative to a reference center (defined by $\vec{S}$ ) is taken to have a Gaussian form characterized by a size parameter, $b$,

$$
\phi(\vec{r}-\vec{S})=\left(\frac{1}{\pi b^{2}}\right)^{3 / 4} e^{-\frac{1}{2 b^{2}}(\vec{r}-\vec{S})^{2}} .
$$

The values of $m_{q}, m_{s}, b, \alpha_{s}$ and $a_{c}$ are determined by reproducing the $\Delta-N$ mass difference, the nucleon mass, a hyperon mass and by requiring a stability condition. The quark-pion coupling constant $g_{q q \pi}$ is obtained from the nucleon-pion coupling constant by a 
slight $(<10 \%)$ correction to the classic symmetry relation, viz.,

$$
\frac{g_{N N \pi}^{2}}{4 \pi}=\left(M_{N} / m_{q}\right)^{2}\left(\frac{5}{3}\right)^{2} \frac{g_{8}^{2}}{4 \pi} e^{m_{\pi}^{2} b^{2} / 2}
$$

where $M_{N}$ is the nucleon mass and the last factor provides the correction due to the extent of the quark wavefunction in the nucleon. The color screening parameter, $\mu$, has been determined by matching our calculation to the mass of the deuteron. All of the parameters are listed in Table I.

Table I: Model Parameters

\begin{tabular}{ccccccc}
\hline \hline$m_{q}, m_{s}(\mathrm{MeV})$ & $b(\mathrm{fm})$ & $a_{c}\left(\mathrm{MeV} \cdot \mathrm{fm}^{-2}\right)$ & $\alpha_{s}$ & $\frac{g_{8}^{2}}{4 \pi}$ & $r_{0}(\mathrm{fm})$ & $\mu\left(f m^{-2}\right)$ \\
\hline 313,634 & 0.6015 & 25.14 & 1.5585 & 0.5926 & 0.8 & 0.85 \\
\hline \hline
\end{tabular}

The model masses of all octet and decuplet baryons are listed in Table II.

Table II: Single Baryon Masses in Units of MeV

\begin{tabular}{ccccccccc}
\hline \hline & $\mathrm{N}$ & $\Sigma$ & $\Lambda$ & $\Xi$ & $\Delta$ & $\Sigma^{*}$ & $\Xi^{*}$ & $\Omega$ \\
\hline theor. & 939.0 & 1210.6 & 1113.6 & 1350.0 & 1232.0 & 1358.1 & 1497.5 & 1650.1 \\
expt. & 939 & 1193 & 1116 & 1318 & 1232 & 1385 & 1533 & 1672 \\
\hline \hline
\end{tabular}

We use the resonating group method to carry out a dynamical calculation. Introducing Gaussian functions with different reference centers $S_{i} \mathrm{i}=1 \ldots \mathrm{n}$, which play the role of generating coordinates in this formalism, to expand the relative motion wave function of the two quark clusters, we have

$$
\chi(\vec{R})=\left(\frac{3}{2 \pi b^{2}}\right)^{3 / 4} \sum_{i} C_{i} e^{-\frac{3}{4}\left(\vec{R}-\vec{S}_{i}\right)^{2} / b^{2}} .
$$

In principle, any set of base wave functions can be used to expand the relative motion wave function. The choice of a Gaussian with the same size parameter, $b$, as the single quark wave function given in Eq.(2), however, allows us to rewrite the resonating group wave function as a product of single quark wave functions; (see Eq.(4) below). This cluster wave function (physical basis) can be expressed in terms of the symmetry basis, classified by the symmetry properties, in a group chain which in turn allows the use of group theory methods to simplify the calculation of the matrix elements of the six quark Hamiltonian 35]. In our calculations, we typically use 12 Gaussian functions to expand the relative motion wave function over the range $0-8 \mathrm{fm}$. For a few channels, such as the deuteron and $H$ particle, 20 Gaussian functions are used to extend the range to $12 \mathrm{fm}$. 
After including the wave function for the center-of-mass motion, the ansatz for the twocluster wave function used in the RGM can be written as

$$
\begin{aligned}
\Psi_{6 q}= & \mathcal{A} \sum_{k} \sum_{i=1}^{n} C_{k, i} \int d \Omega_{S_{i}} \prod_{\alpha=1}^{3} \psi_{R}\left(\vec{r}_{\alpha}, \vec{S}_{i}, \epsilon\right) \prod_{\beta=4}^{6} \psi_{L}\left(\vec{r}_{\beta}, \vec{S}_{i}, \epsilon\right) \\
& {\left[\eta_{I_{1 k} S_{1 k}}\left(B_{1 k}\right) \eta_{I_{2 k} S_{2 k}}\left(B_{2 k}\right)\right]^{I, J=S}\left[\chi_{c}\left(B_{1}\right) \chi_{c}\left(B_{2}\right)\right]^{[\sigma]}, }
\end{aligned}
$$

where $k$ is the channel index. For example, for $S I J=-2,0,0$, we have $k=1,2,3$, corresponding to the channels $\Lambda \Lambda, \mathrm{N} \Xi$ and $\Sigma \Sigma$.

The delocalized orbital wavefunctions, $\psi_{R}\left(\vec{r}, \vec{S}_{i}, \epsilon\right)$ and $\psi_{L}\left(\vec{r}, \overrightarrow{S_{i}}, \epsilon\right)$, are given by

$$
\begin{aligned}
\psi_{R}\left(\vec{r}, \vec{S}_{i}, \epsilon\right) & =\frac{1}{N(\epsilon)}\left(\phi\left(\vec{r}-\frac{\vec{S}_{i}}{2}\right)+\epsilon \phi\left(\vec{r}+\frac{\vec{S}_{i}}{2}\right)\right), \\
\psi_{L}\left(\vec{r}, \vec{S}_{i}, \epsilon\right) & =\frac{1}{N(\epsilon)}\left(\phi\left(\vec{r}+\frac{\vec{S}_{i}}{2}\right)+\epsilon \phi\left(\vec{r}-\frac{\vec{S}_{i}}{2}\right)\right), \\
N(\epsilon) & =\sqrt{1+\epsilon^{2}+2 \epsilon e^{-S_{i}^{2} / 4 b^{2}}}
\end{aligned}
$$

where $\phi\left(\vec{r}-\frac{\overrightarrow{S_{i}}}{2}\right)$ and $\phi\left(\vec{r}+\frac{\overrightarrow{S_{i}}}{2}\right)$ are the single-particle Gaussian quark wave functions referred to above in Eq.(2), with different reference centers $\frac{S_{i}}{2}$ and $-\frac{S_{i}}{2}$, respectively. The delocalization parameter, $\epsilon$, is determined by the dynamics of the quark system rather than being treated as an adjustable parameter.

The initial RGM equation is

$$
\int H\left(\vec{R}, \overrightarrow{R^{\prime}}\right) \chi\left(\overrightarrow{R^{\prime}}\right) d \overrightarrow{R^{\prime}}=E \int N\left(\vec{R}, \overrightarrow{R^{\prime}}\right) \chi\left(\overrightarrow{R^{\prime}}\right) d \overrightarrow{R^{\prime}}
$$

With the above ansatz, the RGM Eq.(6) is converted into an algebraic eigenvalue equation,

$$
\sum_{j, k^{\prime}} C_{j, k^{\prime}} H_{i, j}^{k, k^{\prime}}=E \sum_{j} C_{j, k} N_{i, j}^{k}
$$

where $N_{i, j}^{k}, H_{i, j}^{k, k^{\prime}}$ are the wave function overlaps and Hamiltonian matrix elements, respectively, obtained for the wave functions of Eq.(41).

\section{RESULTS AND DISCUSSION}

Previously, we chose the di- $\Omega$ as an example to study whether or not our model results were sensitive to the meson-exchange cut-off parameter, $r_{0}$, and the result demonstrates that they are not [36]. Hence, we consider it sufficient to calculate six-quark systems of different 
strangeness, $S=-2,-3,-4,-5$, with a representative cutoff value of $r_{0}=0.8 f m$. Table III displays the masses (in $\mathrm{MeV}$ ) calculated for the strange dibaryon states of interest here. The lowest (without taking tensor coupling into account) channel for each SIJ is identified by bold lettering; sc and $c c$ denote single channel (the lowest one) and coupled channels, respectively. '*' denotes those states without coupled channels. Additionally, it should be noted that in our calculation we assume the wavefunction to be zero at the boundary point, which is the usual boundary condition for bound states. If the state is unbound, we will not obtain a stable minimum eigenenergy in the course of extending the boundary point. The unbound states are denoted by "_" in Table III.

Table III : Masses of Six-Quark Systems with Strangeness

\begin{tabular}{|c|c|c|c|}
\hline $\mathrm{S}, \mathrm{I}, \mathrm{J}$ & coupling channels & $\operatorname{Mass}_{s c}$ & $\operatorname{Mass}_{c c}$ \\
\hline$-2,0,0$ & $\boldsymbol{\Lambda} \boldsymbol{\Lambda}-N \Xi-\Sigma \Sigma$ & - & 2225.5 \\
\hline$-3,1 / 2,2$ & $\mathbf{N} \Omega-\Sigma \Xi^{*}-\Xi \Sigma^{*}-\Xi^{*} \Lambda-\Xi^{*} \Sigma^{*}$ & 2566.4 & 2549.1 \\
\hline$-3,1 / 2,1$ & $-N \Omega-\Lambda \Xi^{*}-\Xi \Sigma^{*}-\Sigma^{*} \Xi^{*}-\Sigma \Xi^{*}-\Sigma \Xi$ & - & - \\
\hline$-4,1,0$ & $\Xi \Xi-\Sigma^{*} \Omega-\Xi^{*} \Xi^{*}$ & - & - \\
\hline$-4,0,1$ & $\Xi \Xi-\Lambda \Omega-\Xi \Xi^{*}-\Xi^{*} \Xi^{*}$ & - & - \\
\hline$-5,1 / 2,0$ & $\Xi^{*} \Omega$ & 3145.0 & $*$ \\
\hline$-5,1 / 2,1$ & $\Xi \Omega-\Xi^{*} \Omega$ & - & - \\
\hline$-6,0,0$ & $\Omega \Omega$ & 3298.2 & $*$ \\
\hline
\end{tabular}

In 1977, Jaffe 23] studied the color-magnetic interaction of the one-gluon exchange potential in the multiquark system and found that the most attractive channel is the flavor singlet with quark content $u^{2} d^{2} s^{2}$. Moreover, the same symmetry analysis of the chiral boson exchange potential also leads to the very same conclusion 22].

However dibaryon physics can be very delicate. The deuteron channel is not a channel with strong attraction in any baryon interaction model. If the deuteron had not been found experimentally, it seems highly unlikely that any model would have been able to predict it to be a stable dibaryon. The $H$-particle $(S I J=-200)$ is a six quark state consisting mainly of octet-baryons, similar to the deuteron, and we find only a weak attraction there in our model also. Hence, a qualitative analysis is insufficient to judge whether or not the $H$-particle is strong interaction stable. Systematically, we find that a strong attraction develops only in decuplet-decuplet channels and a mild attraction in octet-decuplet channels. 
Moreover, in the $H$-particle case, the channel coupling effect may even be more important than the deuteron case. In fact, it is not bound without taking coupled channels into account. In our calculation, three channels have been taken into account. These are: $\Sigma \Sigma, N \Xi$ and $\Lambda \Lambda$. The relative motion wave functions of each channel contribution to the $H$-particle are shown in Fig 1. We find that the $\Lambda \Lambda$ channel provides the largest contribution (67\%), followed by the $\mathrm{N} \Xi$ channel (23\%); the $\Sigma \Sigma$ channel contributes only (10\%). However, according to the analysis by Jaffe, the biggest contribution is the $N \Xi$ channel, and the $\Lambda \Lambda$ channel provides the smallest contribution.

The lowest mass we find for the $u^{2} d^{2} s^{2}$ system is $2225.5 \mathrm{MeV}$, which is $6 \mathrm{MeV}$ lower than the experimental threshold of $\Lambda \Lambda$ and $1.7 \mathrm{MeV}$ lower than our model threshold. These values are smaller than the rms uncertainty that may be inferred from our fit to the baryon octet and decuplet in Table II. Furthermore, these values are on the order of corrections one would expect from isospin violations which we have not included. Hence, we can draw no definite conclusion as to whether or not the $H$-particle is strong interaction stable in our model and we consider this to be consistent with recent experimental findings [37].

Besides the binding energy of the $H$, an interesting question regarding the $H$ is its compactness, i.e., whether the $H$ is a compact 6-quark object or a loosely bound $\Lambda \Lambda$ state. Fig.1 indicates that the maxima of the relative motion wave function of the dominant $\Lambda \Lambda$ channel occurs around $1.8 \mathrm{fm}$, and the delocalization parameter, $\epsilon$, of the dominant channel at the maximum is $\sim 0.1$; hence, the $H$ is a loosely bound system similar to the deuteron in our model. Such a similarity may well be more physically sensible than a compact 6-quark structure.

For systems with strangeness $S=-3$, we have calculated the state $\mathrm{N} \Omega$ (SIJ=-3,1/2,2), which was shown to be mildly attractive, with energy below $\Lambda \Xi \pi$ threshold 29]. That conclusion was challenged by Oka [38] and supported by Silvestre-Brac and Leandri [39].

We have carried out a dynamical channel coupling calculation to examine this state further. The $N \Omega, \Lambda \Xi^{*}, \Xi \Sigma^{*}, \Sigma \Xi^{*}, \Xi^{*} \Sigma^{*}$ channels are all included. We find the eigenenergy to be $2549.1 \mathrm{MeV}, 24(54) \mathrm{MeV}$ lower than the $\Lambda \Xi \pi$ experimental(model) threshold. Relative motion cluster wave functions for the individual channels are shown in Fig. 2. The N $\Omega$ channel is by far the dominant one $(77 \%)$ and the maximum for its relative motion wave function occurs at around $0.8 \mathrm{fm}$. The value of $\epsilon$ at this separation is 0.46 . Mixing into the other channels is small. Hence, we find this to be a compact six quark state. 
Note that the D-wave $\Lambda \Xi$ and $\Sigma \Xi$ channels have not been included since no tensor interaction has been included in this calculation. This coupling should be weak because there is no $\pi$ exchange in these channels and $\mathrm{K}$ and $\eta$ exchanges are not important in our model (see next section). Its effect on the eigenenergy of the $N \Omega(\mathrm{SIJ}=-3,1 / 2,2)$ state should be small and the D-wave decay widths to $\Lambda \Xi$ and $\Sigma \Xi$ final states should be small also. Therefore we expect the $S I J=-3,1 / 2,2 N \Omega$ should be a narrow dibaryon resonance. The tensor coupling calculation is currently in progress.

We have also calculated the state $S I J=-3,1 / 2,1$. In the course of extending the boundary point, the lowest eigenenergy closely approaches the model threshold but fails to come to a stable value within our limits of computation for the extension. This result is unchanged by taking into account all possible coupling channels. Due to the fact that there are only weak attractions in our model for the octet-octet channels and the size of the model uncertainty, it is difficult to conclude whether or not there are strong interaction stable states in these channels. We can only conclude that we have not found evidence for a strong interaction stable state with $S I J=-3,1 / 2,1$.

For systems with $\mathrm{S}=-4$, we take the quantum numbers $S I J=-4,1,0$ as an example, because this case bears a number of similarities to the deuteron. For example, in both cases, the lowest mass channel is composed of two octet baryons from the same isodoublet. Also, the matrix element $P_{36}^{s f c}$ characterizing the symmetry property of the system is $-1 / 81$ for both cases. $\left(P_{36}^{s f c}\right.$ is the permutation operator of the quarks between two clusters acting in spin, flavor and color space.) The result shows that the system with $\mathrm{S}=-4, \mathrm{I}=1, \mathrm{~J}=0$ is unbound, even when the $\Xi^{*} \Xi^{*}$ and $\Sigma^{*} \Omega$ channel couplings are taken into account. Because the calculated energy is again very close to the $\Xi \Xi$ threshold this conclusion should be viewed as tentative.

For comparison, we have also calculated the $S I J=-4,0,1$ state as shown in Table III. The $\Xi \Xi, \Xi \Xi^{*}, \Lambda \Omega$ and $\Xi^{*} \Xi^{*}$ coupling channels are included. Our model result is very similar to the $S I J=-4,1,0$, i.e., we do not find a bound state in this channel. The ${ }^{3} S_{1}-{ }^{3} D_{1}$ tensor coupling should be small in the $\Xi \Xi$ channel so taking into account the tensor coupling will not change the unbound character.

For the system with $\mathrm{S}=-5$, we take the $S I J=-5,1 / 2,0$ state as an example. This state is interesting due to its $\left\langle P_{36}^{s f c}\right\rangle$ value, $\sim-1 / 9$, which makes it a Pauli principle favored state. If only two-baryon S-wave channels are taken into account, there is only one channel for this 
state. Our calculation shows that the contribution of the kinetic energy term, due to quark exchange and delocalization effects, contributes strongly towards the formation of a bound state. However, the one-gluon-exchange interaction largely compensates for this attraction and produces a mass of $3145.0 \mathrm{MeV}$, which is $59 \mathrm{MeV}$ lower than the experimental value of the $\Xi^{*} \Omega$ threshold but only about $2 \mathrm{MeV}$ lower than the model threshold. (The one-gluonexchange effect here is quite different from that in the $d^{*}$ case, where large delocalization is favored for a wide range of cluster separations as well as there being a strong effective attraction due to the large reduction in the kinetic energy that accompanies significant delocalization.) We conclude that this state is not a good candidate for a dibaryon resonance search due to its small binding and its $\Xi^{*} \Omega$ content.

Inclusion of the tensor interaction will mix the spin-2 D-wave $\Xi \Omega$ channel with the spin-0 S-wave $\Xi^{*} \Omega$ channel. The tensor coupling should be weak also and we expect that its effect on the $S I J=-5,1 / 2,0$ state is small.

In the same strangeness sector, we also calculated the $S I J=-5,1 / 2,1$ state, since it includes the lowest channel, $\Xi \Omega$. The calculated energy is very close to the $\Xi \Omega$ threshold but a little higher so again there is no bound state, with $S I J=-5,1 / 2,1$, in our model.

To sum up, there are only a few high strangeness states worthy of experimental searches in our model. These are the $H$ particle, the $\mathrm{N} \Omega$ and the di- $\Omega$. The di- $\Omega$ was previously reported in Ref.([36]) and the result is included in Table III.

The $H$-particle and the di- $\Omega$ may be strong interaction stable. However in our model, the binding energies of both are small relative to the model uncertainty. The di- $\Omega$ mass is about $47 \mathrm{MeV}$ lower than the experimental $\Omega \Omega$ threshold. However our model mass for the $\Omega$ is $1650 \mathrm{MeV}$. If this model mass of $\Omega$ were used to calculate the threshold, then the di- $\Omega$ mass is no more than $2 \mathrm{MeV}$ below that threshold. Since our model mass for the single $\Omega$ baryon deviates from the experimental value about $22 \mathrm{MeV}$, a reasonable estimate of the model uncertainty for the dibaryon would be at least that large. Therefore the di- $\Omega$ should not be claimed as a strong interaction stable dibaryon within the model. Similarly, we cannot claim that the $H$-particle is strong interaction stable either. The $S I J=-3,1 / 2,2$ $\mathrm{N} \Omega$ case is certainly not strong interaction stable. However, the state is also certainly lower in mass than the $\mathrm{N} \Omega$ threshold, and quite possibly lower than the $\Lambda \Xi \pi$ threshold, as well. The tensor coupling to the $\Lambda \Xi$ and $\Sigma \Xi$ channels should be weak and the decay width should be small. This strongly suggests that it is a promising candidate for a narrow dibaryon 
resonance. This prediction can be tested by relativistic heavy ion reactions using RHIC detectors through the reconstruction of the vertex mass of the two body decay products, $\Lambda$ and $\Xi$.

\section{THE $K, \eta$ MESON EXCHANGE EFFECT IN THE EXTENDED QDCSM}

The effects of $\mathrm{K}$ and $\eta$ meson exchange have been studied in the di- $\Omega$ channel and found to be negligible in our model [36]. In this section, we carry out a further systematic study of the effect of $(K, \eta)$ meson exchange on the masses of strange dibaryon candidates. A flavorsymmetric octet meson-quark coupling is assumed for all of the octet mesons $(\pi, K, \eta)$. The quark-meson exchange potential has the usual pseudoscalar meson exchange form except for a short range cut-off,

$$
\begin{gathered}
V_{K}\left(r_{i j}\right)=\sum_{a=4}^{7} \theta\left(r-r_{0}\right) \frac{g_{8}^{2}}{4 \pi} \frac{m_{K}^{2}}{12 m_{i} m_{j}} \frac{1}{r_{i j}} e^{-m_{K} r_{i j}} \vec{\sigma}_{i} \cdot \vec{\sigma}_{j} \vec{\lambda}_{i}^{f, a} \cdot \vec{\lambda}_{j}^{f, a}, \\
V_{\eta}\left(r_{i j}\right)=\theta\left(r-r_{0}\right) \frac{g_{8}^{2}}{4 \pi} \frac{m_{\eta}^{2}}{12 m_{i} m_{j}} \frac{1}{r_{i j}} e^{-m_{\eta} r_{i j}} \vec{\sigma}_{i} \cdot \vec{\sigma}_{j} \vec{\lambda}_{i}^{f, 8} \cdot \vec{\lambda}_{j}^{f, 8} .
\end{gathered}
$$

A unified cutoff of $r_{0}=0.8 \mathrm{fm}$ is used. This is aimed at avoiding double counting since, in our model approach[14], short and intermediate range interactions have been accounted for by the combination of quark delocalization and color screening. The model parameters $\left(b, a_{c}, \alpha_{s}\right)$ are refitted as before, and are listed in Table IV. The recalculated masses of octet and decuplet baryons are listed in Table V.

Table IV: Model Parameters

\begin{tabular}{ccccccc}
\hline \hline$m_{q}, m_{s}(\mathrm{MeV})$ & $b(\mathrm{fm})$ & $a_{c}\left(\mathrm{MeV} \cdot \mathrm{fm}^{-2}\right)$ & $\alpha_{s}$ & $\frac{g_{8}^{2}}{4 \pi}$ & $r_{0}(\mathrm{fm})$ & $\mu\left(\mathrm{fm}^{-2}\right)$ \\
\hline 313,634 & 0.6022 & 25.03 & 1.5547 & 0.5926 & 0.8 & 0.90 \\
\hline \hline
\end{tabular}

Table V: Single Baryon Masses in Units of MeV

\begin{tabular}{ccccccccc}
\hline \hline & $\mathrm{N}$ & $\Sigma$ & $\Lambda$ & $\Xi$ & $\Delta$ & $\Sigma^{*}$ & $\Xi^{*}$ & $\Omega$ \\
\hline theor. & 939.0 & 1217.47 & 1116.90 & 1357.56 & 1232.0 & 1359.61 & 1499.70 & 1652.27 \\
expt. & 939 & 1193 & 1116 & 1318 & 1232 & 1385 & 1533 & 1672 \\
\hline \hline
\end{tabular}

Comparing Tables I,II and IV,V, it is apparent that the addition of $\mathrm{K}$ and $\eta$ exchanges has modified the model parameters and single baryon masses only slightly. We also find that 
the properties of the deuteron are reproduced as well as before by a minor readjustment of the color-screening parameter $\mu$ 36].

With the full octet meson exchange, we recalculated every single channel case with different quantum numbers. For the $H$-particle, we calculated both the single channel and coupled channels, because it is very sensitive to channel coupling. The results are presented in Tables VI and VII. For comparison, the results with $\pi$ exchange only are also listed.

Table VI : Masses of Six-Quark Systems with $\pi$ exchange only and full octet pseudoscalar meson exchange. The masses are given in $\mathrm{MeV}$.

\begin{tabular}{cccc}
\hline \hline S,I,J & single channel & only $\pi$ exchange & $\pi, K, \eta$ exchange \\
\hline$-3,1 / 2,2$ & $N \Omega$ & 2566.37 & 2556.95 \\
$-3,1 / 2,1$ & $\Lambda \Xi$ & - & - \\
$-4,1,0$ & $\Xi \Xi$ & - & - \\
$-4,0,1$ & $\Xi \Xi$ & - & - \\
$-5,1 / 2,1$ & $\Xi \Omega$ & - & - \\
$-5,1 / 2,0$ & $\Xi^{*} \Omega$ & 3145.01 & 3146.15 \\
$-6,0,0$ & $\Omega \Omega$ & 3298.20 & 3300.00 \\
\hline \hline
\end{tabular}

Table VII : Mass of $H$-particle $(S I J=-2,0,0)$ with $\pi$ exchange only and full octet

\begin{tabular}{lccc}
\multicolumn{4}{c}{ pseudoscalar meson exchange. The masses are given in $\mathrm{MeV}}$. \\
\hline \hline \multirow{4}{*}{ single channels } & $\Sigma \Sigma$ & only $\pi$ exchange & $\pi, K, \eta$ exchange \\
\hline & $N \Xi$ & 2280.40 & 2282.67 \\
& $\Lambda \Lambda$ & - & 2268.12 \\
\hline coupled channels $\Sigma \Sigma-N \Xi-\Lambda \Lambda$ & 2225.48 & - \\
\hline \hline
\end{tabular}

Tables VI and VII show that those systems which are unbound in the extended QDCSM with $\pi$ exchange only, such as $S I J=-3,1 / 2,1 ;-4,1,0 ;-4,0,1$ and $-5,1 / 2,1$, remain unbound after adding $K$ and $\eta$ exchanges, while those systems, which are bound in Table III, remain bound. Moreover, their masses are almost unaffected by the addition of $K$ and $\eta$ exchange. The largest difference is not more than $10 \mathrm{MeV}$. Especially for the $H$-particle, the $\Lambda \Lambda$ channel would be unbound without taking into account the $N \Xi$ and $\Sigma \Sigma$ channel coupling; this character is not affected either by adding $K$ and $\eta$ exchange. Even the $\Lambda \Lambda$, $N \Xi$ and $\Sigma \Sigma$ channel mixing fractions, $56 \%, 23 \%$ and $21 \%$, respectively, are very similar to 
the case with $\pi$ exchange only. All of these results confirm our expectation that heavier meson $(K, \eta)$ exchange has already been mostly accounted for by the quark delocalization and color screening effects in our model approach. Hence, explicit inclusion of $K$ and $\eta$ exchanges beyond the cutoff scale is not important in our approach.

\section{FURTHER DISCUSSION ABOUT THE MECHANISM OF INTERMEDIATE RANGE ATTRACTION}

We have reported that the QDCSM gives very similar effective BB interactions to other models [14], in general. However a careful comparison found that our model results for the binding energy of high strangeness dibaryons are systematically smaller than those of the chiral quark model[40]. The difference is mainly due to the different mechanism for the effective BB intermediate range attraction.

In the chiral quark model 8, 9, 40] the intermediate range attraction is attributed to $\sigma$ meson exchange. Because of its scalar-isoscalar character, $\sigma$ meson exchange provides a universal attraction independent of the flavor.

In the Bonn meson exchange model 41] the $\sigma$ meson is an effective description of correlated two $\pi$ exchange. This point has been confirmed by a chiral perturbation calculation of the NN interaction 42]. Such an effective $\sigma$-N coupling can not be extended from the NN channel to other channels with strangeness by a universal $\sigma$-baryon coupling, because in the NN channel, there are $\mathrm{NN}, \mathrm{N} \Delta$ and $\Delta \Delta$ intermediate states for two $\pi$ exchange, while for the $\Lambda \Lambda$ channel, there is only the $\Sigma \Sigma$ intermediate state, and for the $\Omega \Omega$ channel, no such intermediate state is possible. Therefore it is not justified to fix the parameters of $\sigma$ exchange in the NN channel and then directly extend that exchange to channels with strangeness.

There are arguments based on spontaneous chiral symmetry breaking for introduction of $\sigma$ meson quark coupling. In the $\mathrm{SU}(2)$ case, the non-linear realization of chiral symmetry can be linearized and in turn the $\sigma$ and $\pi$ meson coupling constants can be unified [7, 8]. However one should note this universal coupling is restricted to the $u$ and $d$ quarks and the effective $\sigma$ is still due to even number multiple $\pi$ exchange. In the $\mathrm{SU}(3)$ case, one can introduce $\mathrm{SU}(3)$ chiral symmetry by neglecting the difference between the $s$ and light quark masses, followed by spontaneous chiral symmetry breaking. However, the nonlinear realization of $\mathrm{SU}(3)$ can not be linearized in the same way as in the $\mathrm{SU}(2)$ case to obtain 
a universal $u, d$ and $s$ quark $\sigma$ coupling. The internal quark structure of the $\sigma$ meson is a controversial issue, but an equal mix of $u \bar{u}+d \bar{d}+s \bar{s}$ is quite unexpected and so also is a universal $u, d$ and $s$ quark $\sigma$ coupling. Hence, a strong attraction in high strangeness channels arising from such a universal $\sigma$ quark coupling is quite questionable also.

In the QDCSM, quark delocalization and color screening work together to provide appropriate short-range repulsion and intermediate-range attraction for different channels. We illustrate this mechanism by showing contributions of the kinetic energy, confinement, color Coulomb, and color magnetic terms to the effective BB potential $V_{B B}(S)$, as well as the total sum, in curves a-e, respectively in Figs.3-6 for a few typical channels. The value of $\epsilon$ varies with the separation $S$ and is also listed in Figs.3-6. The contribution of $\pi, K, \eta$ exchange with a cut-off $r_{0}=0.8 \mathrm{fm}$ is small so we do not show it. These figures are ordered in terms of increasing values of $\left\langle P_{36}^{s f c}\right\rangle$, from Pauli favored to Pauli forbidden.

The effective baryon-baryon interactions shown in these figures are obtained in the BornOppenheimer approximation,

$$
V_{B B}\left(S_{i}\right)=\frac{\left\langle\Psi_{B B}\left(S_{i}\right)|H| \Psi_{B B}\left(S_{i}\right)\right\rangle}{\left\langle\Psi_{B B}\left(S_{i}\right) \mid \Psi_{B B}\left(S_{i}\right)\right\rangle}-\left.\frac{\left\langle\Psi_{B B}\left(S_{i}\right)|H| \Psi_{B B}\left(S_{i}\right)\right\rangle}{\left\langle\Psi_{B B}\left(S_{i}\right) \mid \Psi_{B B}\left(S_{i}\right)\right\rangle}\right|_{S_{i} \rightarrow \infty, \epsilon=0} .
$$

Where the $\Psi_{B B}\left(S_{i}\right)$ is the antisymmetric six quark cluster state at a specified separation, $S_{i}$, as given in Eq. (4) without summation over $k$ and $i$; $H$ is the six quark Hamiltonian (1). The contribution of each term in Eq. (1) is defined similarly, for example,

$$
V_{B B}^{\text {conf }}\left(S_{i}\right)=\frac{\left\langle\Psi_{B B}\left(S_{i}\right)\left|V_{\text {conf }}\right| \Psi_{B B}\left(S_{i}\right)\right\rangle}{\left\langle\Psi_{B B}\left(S_{i}\right) \mid \Psi_{B B}\left(S_{i}\right)\right\rangle}-\left.\frac{\left\langle\Psi_{B B}\left(S_{i}\right)\left|V_{\text {conf } f}\right| \Psi_{B B}\right\rangle}{\left\langle\Psi_{B B}\left(S_{i}\right) \mid \Psi_{B B}\left(S_{i}\right)\right\rangle}\right|_{S_{i} \rightarrow \infty, \epsilon=0} .
$$

Quark exchange alone induces a weak reduction in kinetic energy. Quark delocalization enhances this kinetic energy reduction. The kinetic energy reduction is also dependent on the strangeness of the channels due to the inverse quark mass dependence of the quark kinetic energy. The higher the strangeness, the smaller the contribution of quark kinetic energy and hence the smaller the reduction of the kinetic energy due to delocalization (see Figs.3-6(a)). This makes the intermediate range attraction weaker for the higher strangeness channels and is the main reason that our model gives less binding for the $S=-4,-5,-6$ states than does the chiral quark model[40].

If the usual quadratic color confinement is used, it does not contribute to the effective BB interaction. With the introduction of quark delocalization, the usual color confinement contributes an effective repulsion, as shown in Fig.7. The color Coulomb has a similar 
behavior, as shown in Figs.3-6(c); it produces almost no contribution to the effective BB interaction without quark delocalization, and contributes an effective repulsion with quark delocalization. These two terms working together almost totally forbid quark delocalization. This implies that the internal structure of the baryon is unaffected by the mutual interaction. However, that is inconsistent with the observed difference between the nucleon structure function in a nucleus and in isolation as seen in deep inelastic lepton scattering (EMC effect).

On the other hand, there is no compelling reason to assume that the two body confinement potential is a good approximation for a multi-quark system. At the very least, the three gluon and the three body instanton interactions, which do not contribute to the $q \bar{q}$ meson and $q^{3}$ baryon but do contribute to the multi-quark system, have been omitted in every two body confinement model. The color flux scenario revealed in the lattice QCD calculation of two and three quark systems raises questions regarding an additive two body confinement approximation. To take these facts into account, the QDCSM reparametrizes the confinement by introducing color screening [10, 11, 12, 15, 26, 28]. Figs.3-6(b) show that after the introduction of color screening, the confinement term contributes an additional attraction, which mainly reduces the repulsive core of the effective BB interactions (See Fig.7). There is a systematic uncertainty in our model related to this term which is not yet quantified.

The color magnetic term generally contributes to a repulsive core except for a few Pauli favored channels where it contributes an additional intermediate range attraction so that these channels develop a strong effective attraction. It is also dependent on the strangeness of the channels: The higher the channel strangeness, the weaker the color magnetic contribution, due to the inverse strange quark mass in the color magnetic term. Figs.3-6(d) display these results.

Altogether, these figures show that in the QDCSM the intermediate range attraction is mainly due to reduction of the quark kinetic energy, and the degree of reduction of the quark kinetic energy is connected with the degree of quark delocalization; the latter is determined by the competition between these four terms and that competition is different in different channels. We have mentioned before that in the $d^{*}$ channel this competition produces a large quark delocalization for a wide range of separations between two $\Delta$-like quark clusters so that the kinetic energy acquires a correspondingly large reduction, which in turn gives 
rise to a very strong attraction in this channel. (See Fig.3). The $S I J=-6,0,0$ has the

same $\left\langle P_{36}^{s f c}>\right.$ as that of the $S I J=0,0,3$ channel and has a similar opportunity to develop large quark delocalizations and strong attraction. However, there the competition does not allow as large a quark delocalization to develop and so the resulting attraction is not as strong as in the $d^{*}$ channel. (See Fig.4.)

\section{CONCLUSION}

To sum up, we have carried out a dynamical calculation for the most promising dibaryon candidates with high strangeness by using the extended QDCSM. Only a few high strangeness states recommend themselves for experimental searches. These are the $H$ particle, the $\mathrm{N} \Omega$ and the di- $\Omega$. We recommend searching for the $(S I J=-3,1 / 2,2)$ using $N \Omega \rightarrow \Lambda \Xi \mathrm{D}$-wave decay vertex mass reconstruction with the RHIC detectors as the case with the best likelihood.

In the QDCSM, it is the quark dynamics that controls the competition among the four terms in the Hamiltonian (1): the kinetic energy, the confinement, the color Coulomb, and the color magnetic, and which determines the overall effective BB interactions. These are quite similar, though not identical, to those of the quark-gluon-meson hybrid model [14] for the majority of $\mathrm{BB}$ channels. The exchange of explicit $K$ and $\eta$ mesons is not important in our model for mass estimates of strange dibaryons because of our short range cut-off.

This model, which has the fewest parameters, describes the properties of the deuteron and the existing $\mathrm{NN}, \mathrm{N} \Lambda$ and $\mathrm{N} \Sigma$ scattering data. Up to now it is the only model which gives an explanation of the long standing fact that the nuclear and molecular forces are similar in character despite the obvious length and energy scale differences and that nuclei are well described as a collection of $\mathrm{A}$ nucleons rather than $3 \mathrm{~A}$ quarks. In view of the fact that the $H$-particle has not been observed experimentally, the BB interaction in the $\Lambda \Lambda$ channel 37] predicted by this model may be a good approximation of the real world. Further refinement is possible by including more channel couplings and spin-orbit and tensor interactions.

Of course, the QDCSM is only a model of QCD. The high strangeness dibaryon resonances may be a good venue for determining whether the QDCSM mechanism for the intermediate range attraction is more realistic than that of a universal $u, d, s$ quark- $\sigma$ meson coupling.

This work is supported by NSFC contract 90103018 and by the U.S. Department of 
Energy under contract W-7405-ENG-36. F. Wang would like to thank the ITP for their support through the visiting program.

[1] R.T. Cahill and C.D. Roberts, Phys. Rev. D32, 2419 (1985).

[2] P.C. Tandy, Prog. Part. Nucl. Phys. 39, 117 (1997).

R.T. Cahill and S.M. Gunner, Fizika B7, 17 (1998).

X.F. Lu, Y.X. Liu, H.S. Zong and E.G. Zhao, Phys. Rev. C58, 1195 (1998).

[3] C.D. Roberts and S.M. Schmidt, Prog. Part. Nucl. Phys. 45S1, 1-103 (2000).

[4] A. De Rújula, H. Georgi and S.L. Glashow, Phys. Rev. D12, 147 (1975).

[5] N. Isgur and G. Karl, Phys. Rev. D18, 4187 (1978); D19, 2653 (1979); D20, 1191 (1979).

[6] A. Faessler and F. Fernandez, Phys. Lett. B124, 145 (1983).

[7] I.T. Obukhovsky and A.M. Kusainov, Phys. Lett. B238, 142 (1990).

[8] F. Fernandez, A. Valcarce, U. Straub and Z. Faessler, J. Phys. G19, 2013 (1993).

[9] Y. Fujiwara, C. Nakamoto and Y. Suzuki, Phys. Rev. Lett. 76, 2242 (1996).

Y. Fujiwara, T. Fujita, M. Kohno, C. Nakamoto, and Y. Suzuki, Phys. Rev. C65, 014002 (2002).

[10] F. Wang, G.H. Wu, L.J. Teng and T. Goldman, Phys. Rev. Lett. 69, 2901 (1992).

[11] G.H. Wu, L.J. Teng, J.L. Ping, F. Wang and T. Goldman, Phys. Rev. C53, 1161 (1996).

[12] G.H. Wu, J.L. Ping, F. Wang and T. Goldman, Nucl. Phys. A673, 279 (2000).

[13] J.L. Ping, F. Wang and T. Goldman, Phys. Rev. C62, 054007 (2000).

[14] H.R. Pang, J.L. Ping, F. Wang and T. Goldman, Phys. Rev. C65, 014003 (2001).

[15] J.L. Ping, H.R. Pang, F. Wang and T. Goldman, Phys. Rev. C65, 044003 (2002).

[16] X.F. Lu, J.L. Ping and F. Wang, Chin. Phys. Lett. 20, 42 (2003).

[17] L.Ya. Glozman, et al., Phys. Rev. C57, 3406 (1998); Nucl. Phys. A663-664, 103c (2000); nucl-th/9909021

[18] K.F. Liu et al., Phys. Rev. D59, 112001 (1999); Phys. Rev. D61, 118502 (2000).

[19] N. Isgur, Phys. Rev. D61, 118501; D62, 054026 (2000).

[20] H. Collins and H. Georgi, Phys. Rev. D59, 094010 (1999).

[21] A. Valcarce, P. Gonzalez, F. Fernandez and V. Vento, Phys. Rev. C61, 019803 (2000);

H. Garcilazo, A. Valcarce and F. Fernandez, Phys. Rev. C63, 035207 (2001); 
C. Nakamoto and H. Toki, Prog. Theor. Phys. 99, 1001 (1998).

[22] F. Wang, J.L. Ping, H.R. Pang and T. Goldman, Mod. Phys. Lett. A18, 356 (2003); nucl-th/0212012.

[23] R.L. Jaffe, Phys. Rev. Lett. 38, 195 (1977).

[24] R. Bilger, et al., Phys. Rev. Lett. 71, 42 (1993); 72, 2792 (1994); 79, 3849 (1997); Phys. Lett. B420, 37 (1998); B428, 18 (1998); B443, 77 (1998); Phys. Rev. C58, 1576 (1998); Nucl. Phys. A663-664, 469c (2000);

F. Hinterberger et al., Nucl. Phys. A663-664, 533c (2000);

W. Brodowski, et al., Phys. Rev. Lett. 88, 192301 (2002); nucl-ex/0206020,

S.M. Kiselev, et al., Nucl. Phys. A650, 78 (1999).

[25] T. Goldman et al., Phys. Rev. C39, 1889 (1989).

[26] F. Wang et al., Phys. Rev. C51, 3411 (1995).

J.L. Ping, F. Wang and T. Goldman, Nucl. Phys. A657, 95 (1999).

[27] T. Goldman, et al., Mod. Phys. Lett. A13, 59 (1998).

[28] J.L. Ping, F. Wang and T. Goldman, Nucl. Phys. A688, 871 (2001).

[29] T. Goldman et al., Phys. Rev. Lett. 59, 627 (1987).

[30] V.B. Kopeliovich, Nucl. Phys. A639, 75c (1998).

[31] Z.Y. Zhang, Y.W. Yu, C.R. Ching, T.H. Ho and Z.D. Lu, Phys. Rev. C61, 065204 (2000).

[32] P. LaFrance and E.L. Lomon, Phys. Rev. D34, 1341 (1986) and references therein.

[33] F. Lehar, in: Baryons'98, ed. D.W. Menze and B.Ch. Metsch, (World Scientific, Singapore, 1999) p.622.

[34] A.J. Buchmann, Y. Yamauchi and A. Faessler, Nucl. Phys. A496, 621 (1989).

[35] F. Wang, J. L. Ping and T. Goldman, Phys. Rev. C51, 1648 (1995).

[36] H.R. Pang,J.L. Ping,F. Wang and T. Goldman, Commun. Theor. Phys. 38, 424 (2002); Phys. Rev. C66, 025201 (2002).

[37] H. Takahashi et al., Phys. Rev. Lett. 87, 212502 (2002).

[38] M. Oka, Phys. Rev. D38, 298 (1988).

[39] B. Silvestre-Brac and J. Leandri, Phys. Rev. D45, 4221 (1992).

[40] Q.B. Li, P.N. Shen, Z.Y. Zhang and Y.W. Yu, Nucl. Phys. A683, 487 (2001).

[41] R. Machleidt, K. Holinde and Ch. Elster, Phys. Rep. 149, 1 (1987).

[42] D.R. Entem and R. Machleidt, Phys. Rev. C68, 041001 (2003); nucl-th/0304018. 


\section{FIGURE CAPTIONS}

Fig.1 Relative motion wave functions of the coupled channels for the SIJ=-2,0,0 state with eigenenergy $2225.5 \mathrm{MeV}$.

Fig.2 Relative motion wavefunctions of the coupled channels for the SIJ=-3,1/2,2 state with eigenenergy $2549.1 \mathrm{MeV}$.

Fig.3 Contributions of kinetic energy, confinement, color Coulomb and color magnetic terms (a-d respectively) to the effective potential and the total (e) for the SIJ $=0,0,3 \Delta \Delta$ channel with $P_{36}^{s f c}=-1 / 9$. In each subfigure, the dotted curve is for delocalization parameter $\epsilon=0.0$, dashed curve for $\epsilon=0.5$, dashed-dotted curve for $\epsilon=1.0$; the solid curve is for the self-consistent value of $\epsilon$ determined by the system dynamics.

Fig.4 The same as Fig.3 for the state $S I J=-6,0,0 \Omega \Omega$ with $\left\langle P_{36}^{s f c}\right\rangle=-1 / 9$.

Fig.5 The same as Fig.3 for the state $S I J=0,0,1 \mathrm{NN}$ with $\left\langle P_{36}^{s f c}\right\rangle=-1 / 81$.

Fig.6 The same as Fig.3 for the state $S I J=-2,0,0 \Lambda \Lambda$ with $<P_{36}^{s f c}>=0$.

Fig.7 The contribution of conventional quadratic color confinement to the effective BB interaction. The solid curve is for $\epsilon=0.0$, dotted curve for $\epsilon=0.5$ and the dashed curve for $\epsilon=1.0$. 


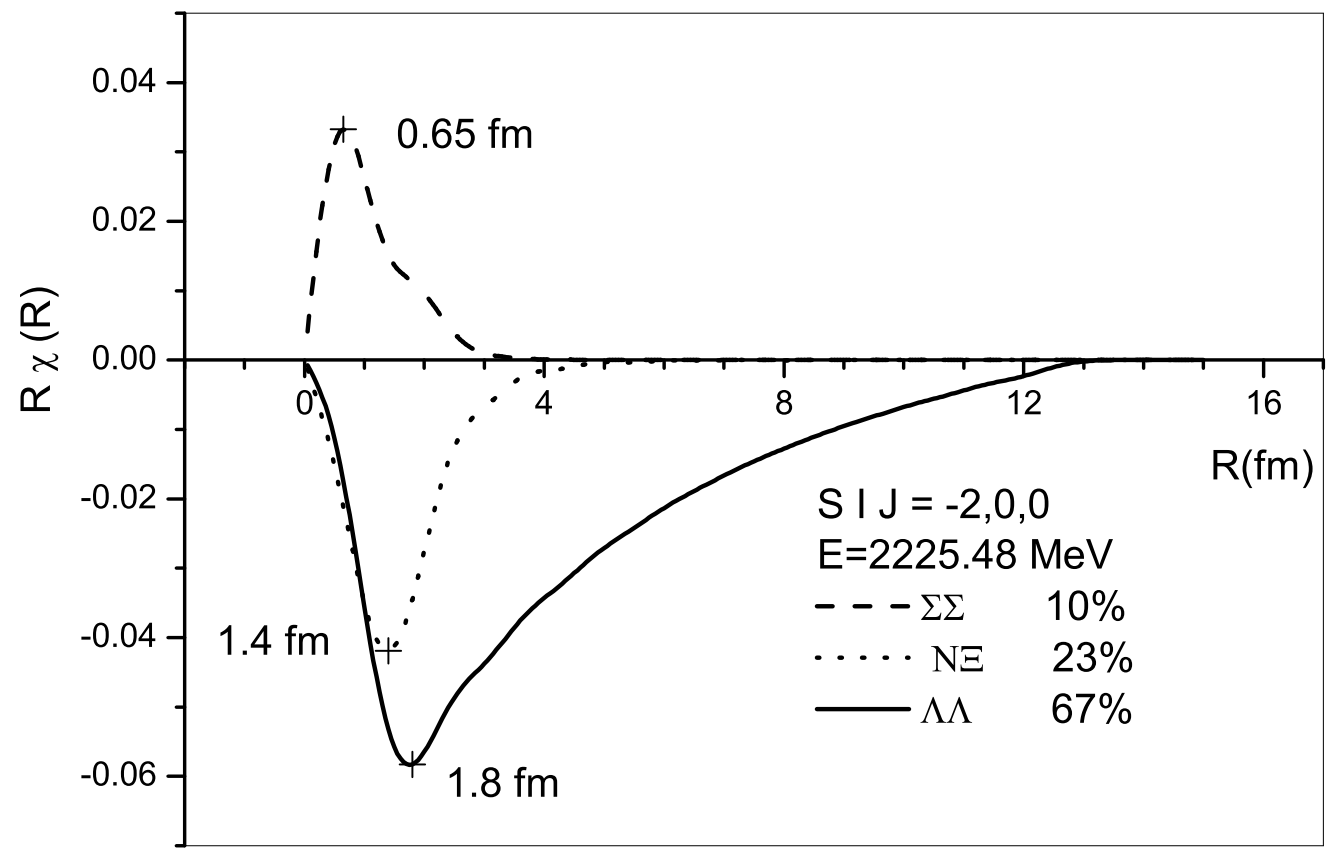

FIG. 1: Relative motion wave functions of the coupled channels for the SIJ=-2,0,0 state with eigenenergy $2225.5 \mathrm{MeV}$. 


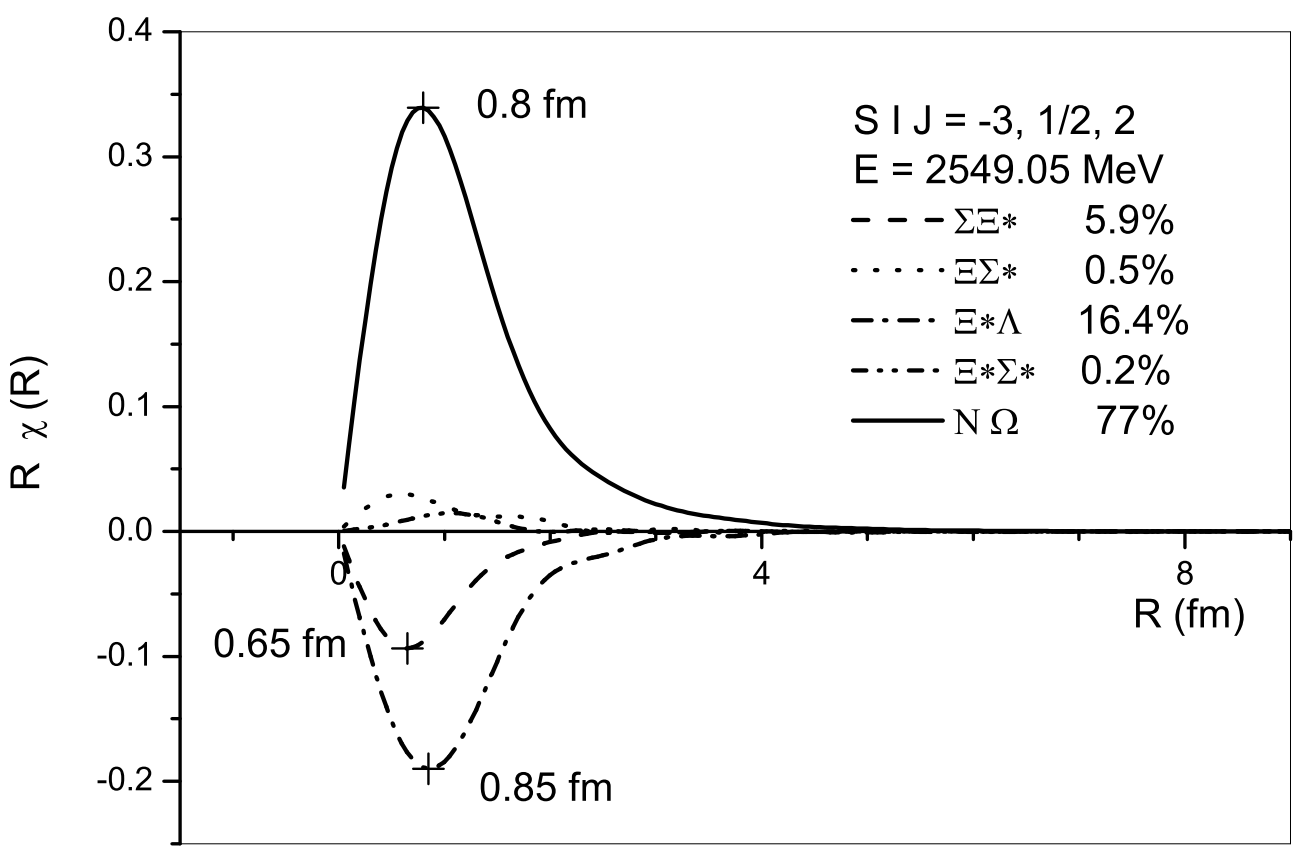

FIG. 2: Relative motion wavefunctions of the coupled channels for the SIJ=-3,1/2,2 state with eigenenergy $2549.1 \mathrm{MeV}$. 


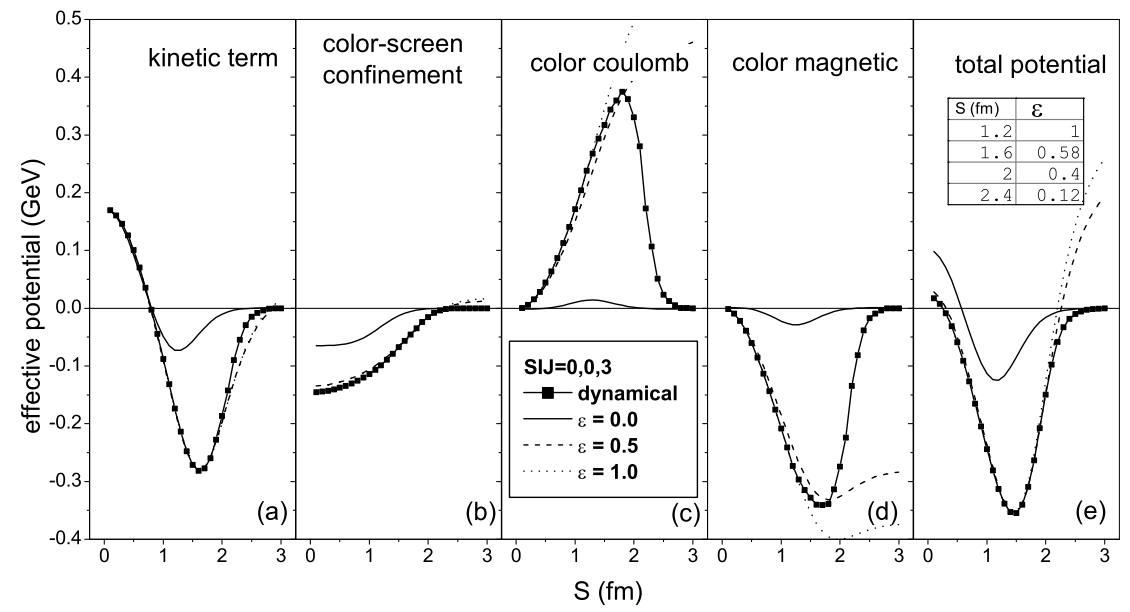

FIG. 3: Contributions of kinetic energy, confinement, color Coulomb and color magnetic terms (a-d respectively) to the effective potential and the total (e) for the SIJ $=0,0,3 \Delta \Delta$ channel with $P_{36}^{s f c}=-1 / 9$. In each subfigure, the dotted curve is for delocalization parameter $\epsilon=0.0$, dashed curve for $\epsilon=0.5$, dashed-dotted curve for $\epsilon=1.0$; the solid curve is for the self-consistent value of $\epsilon$ determined by the system dynamics. 


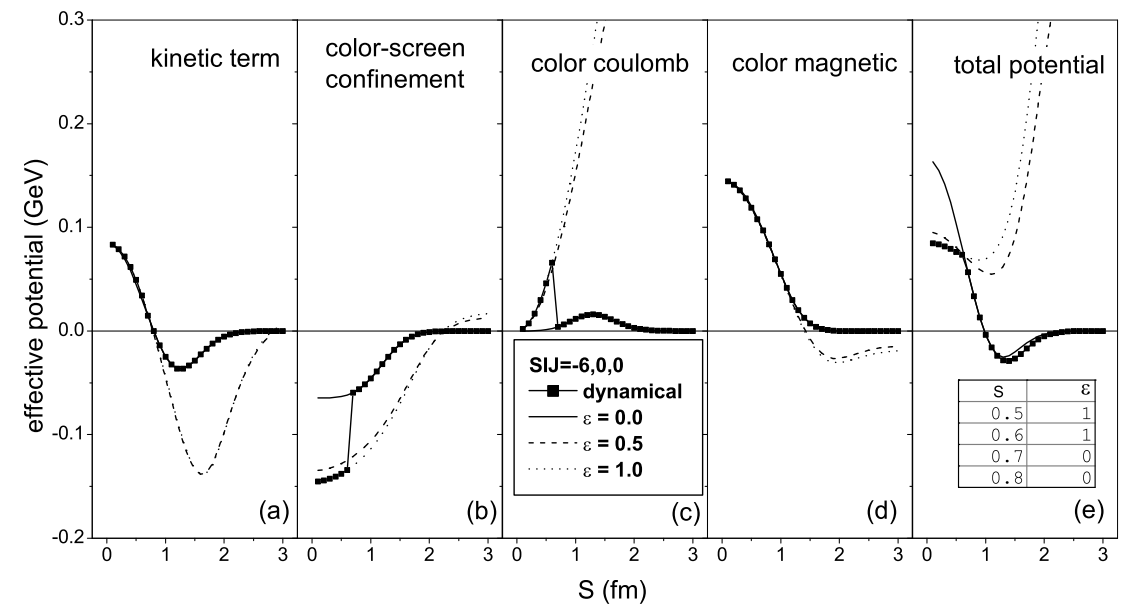

FIG. 4: The same as Fig.3 for the state $S I J=-6,0,0 \Omega \Omega$ with $<P_{36}^{s f c}>=-1 / 9$.

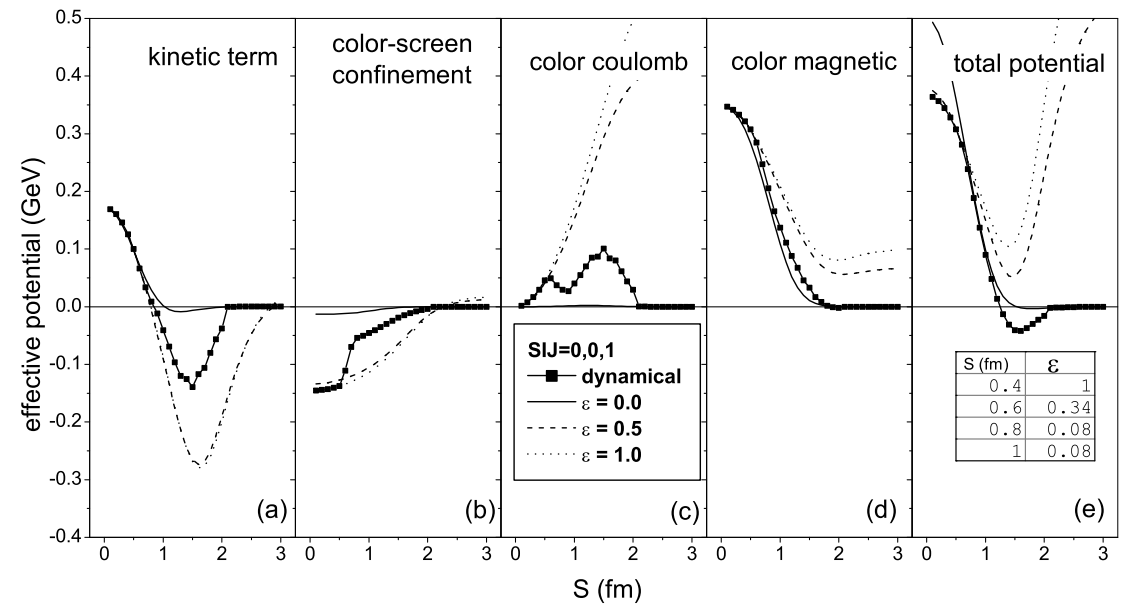

FIG. 5: The same as Fig.3 for the state $S I J=0,0,1 \mathrm{NN}$ with $\left\langle P_{36}^{s f c}\right\rangle=-1 / 81$. 


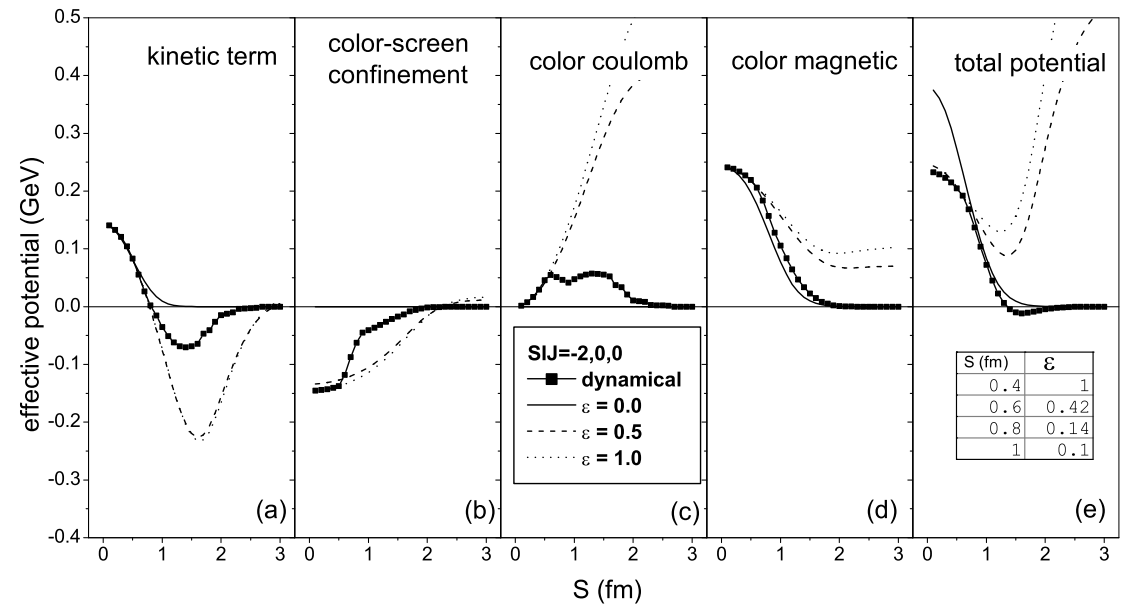

FIG. 6: The same as Fig.3 for the state $S I J=-2,0,0 \Lambda \Lambda$ with $\left\langle P_{36}^{s f c}\right\rangle=0$. 


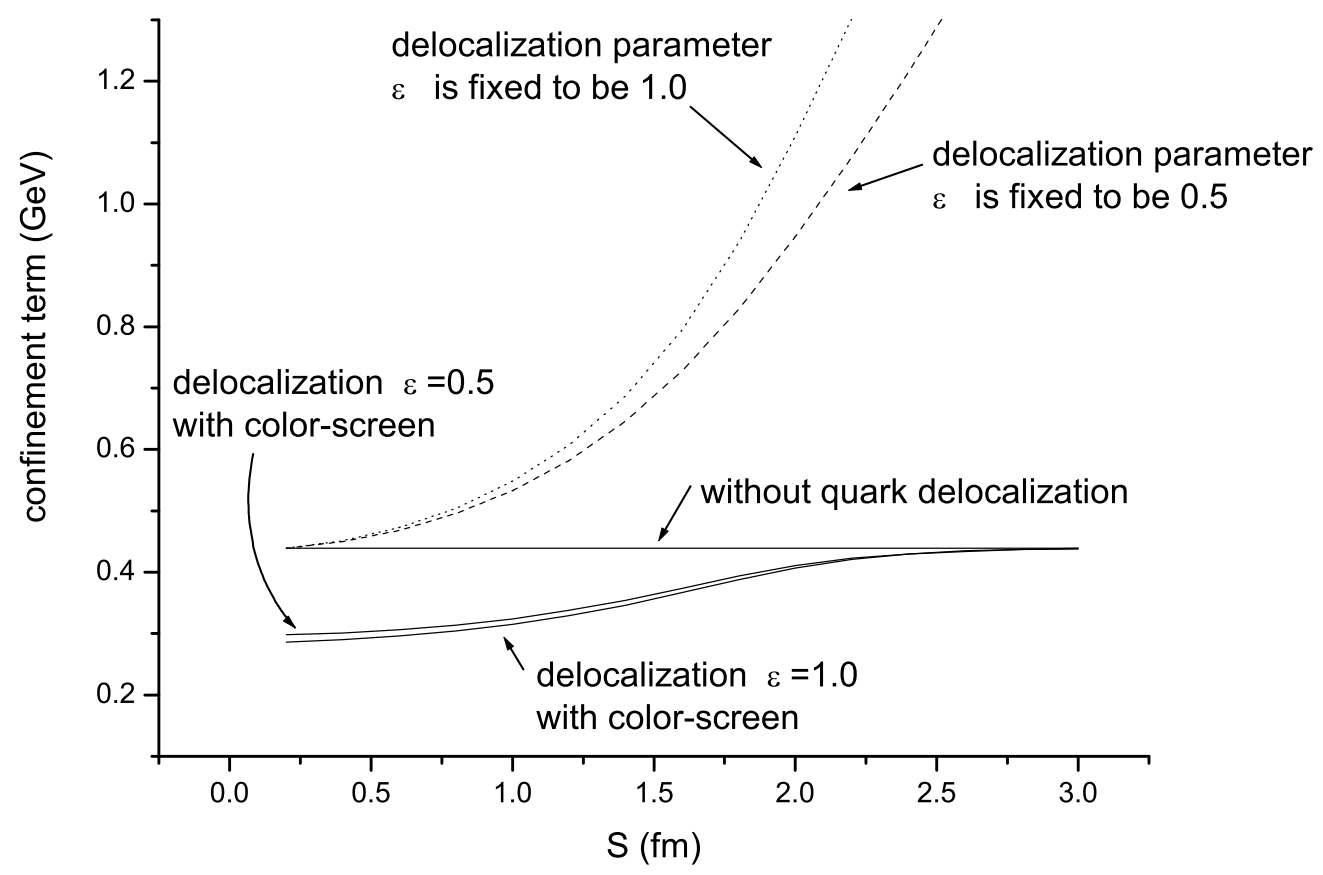

FIG. 7: The contribution of the usual quadratic color confinement to the effective BB interaction with and without quark delocalization and color-screening. 